\title{
Closure of a tyrosine/tryptophan aromatic gate leads to a compact fold in apo flavodoxin
}

Carlos G. Genzor ${ }^{2}$, Aurea Perales-Alcón ${ }^{1}$, Javier Sancho ${ }^{2}$ and Antonio Romero ${ }^{1}$ Nature Structural Biology 3, 329-332 (1996).

Table 1 was misprinted. The version below is as it should have appeared in the April 1996 issue.

Table 1 Details of hydrogen bonds and structure determination

\begin{tabular}{|c|c|c|c|c|c|c|c|c|c|}
\hline Thr 56 & $\mathrm{~N}$ & Thr 88 & $O \gamma^{1}$ & $2.9(\AA)$ & $\mathrm{SO}_{4}$ & 01 & Thr 10 & $O \gamma^{1}$ & $2.8(\AA)$ \\
\hline $\mathrm{SO}_{4}$ & 01 & Thr 15 & $\mathrm{~N}$ & $2.8(\AA ̊)$ & $\mathrm{SO}_{4}^{4}$ & 01 & Thr 15 & $O \gamma^{1}$ & $2.9(\AA)$ \\
\hline $\mathrm{SO}_{4}$ & 02 & Thr 12 & $N$ & $3.2(\AA)$ & $\mathrm{SO}_{4}^{4}$ & 02 & Thr 12 & $O y^{1}$ & $2.7(\AA)$ \\
\hline $\mathrm{SO}_{4}$ & 02 & Lys 14 & $\mathrm{~N}$ & $2.8(\AA)$ & $\mathrm{SO}_{4}$ & 03 & Trp 57 & $N \varepsilon^{1}$ & $3.0(\AA \AA)$ \\
\hline $\mathrm{SO}_{4}$ & 03 & Thr 88 & $O \gamma^{1}$ & $2.8(\AA)$ & $\mathrm{SO}_{4}^{4}$ & 04 & Gln 11 & $\mathrm{~N}$ & $2.9(\AA ̊)$ \\
\hline $\mathrm{SO}_{4}$ & 04 & Thr 12 & $\mathrm{~N}$ & $3.1(\AA)$ & $\mathrm{SO}_{4}$ & 04 & Trp 57 & $N \varepsilon^{1}$ & $3.1(\AA)$ \\
\hline Lys 14 & $\mathrm{~N} \varepsilon$ & Asp 146 & $O \sigma^{1}$ & $3.5(\AA)$ & & & & & \\
\hline
\end{tabular}

b, Data collection and refinement statistics

Data collection

Space group

maximum resolution

$P 2,2,2$,

Number of measured reflections

Number of unique reflections $\quad 8,437$

$R_{\text {merge }}{ }^{1}$

$7.4 \%$

Completeness $(\%)(I>2 \sigma)$

$$
\begin{aligned}
\text { resolution range: } \infty-2.0 \AA & 92 \\
2.1-2.0 \AA & 74
\end{aligned}
$$

Refinement

Resolution range

$6-2 \AA$

Number of protein atoms

1,326

Number of reflections used in the refinement $\quad 7,823$

Crystallographic $R$-factor ${ }^{2}(\%)$

18.2

R.m.s.d. from ideal bond lengths $(\AA)$

0.009

R.m.s.d. from ideal bond angles $\left({ }^{\circ}\right)$

1.482

R.m.s.d. from ideal dihedral angles $\left(^{\circ}\right)$

26.0

R.m.s.d. from ideal improper angles $\left(^{\circ}\right)$

1.371

Average temperature factors $\left(\AA^{2}\right)$

apo

Protein atoms

15.2

peptide segment Pro 55-Leu 62

Trp 57

28.2

Phe 94

13.1

15.2

holo

8.7

5.0

3.3

2.3

${ }^{1} R_{\text {merge }}=\Sigma \mathrm{j}\left(\mid I_{\mathrm{j}}-\langle i>|\right) / \mathrm{\Sigma}<\mathrm{l}, I_{\mathrm{j}}$ is the observed scaled intensity of each unique reflection $\mathrm{j}$ and $\langle l>$ is the mean value of multiple observations.

${ }^{2}$ Crystallographic $R$-factor, $R=\Sigma|| \boldsymbol{F}_{\mathrm{o}}|-| \boldsymbol{F}_{\mathrm{c}}|| \Sigma\left|\boldsymbol{F}_{\mathrm{o}}\right|$. 